\title{
Mixed Co-Ni Carbide Clusters. Part 1. Synthesis and Structural Characterization of the $\left[\mathrm{Co}_{3} \mathrm{Ni}_{9} \mathrm{C}(\mathrm{CO})_{20}\right]^{3-}$ Trianion $\dagger$
}

\author{
Alessandro Ceriotti, Roberto Della Pergola, and Giuliano Longoni * \\ Dipartimento di Chimica Inorganica e Metallorganica, via G. Venezian 21, 20133 Milano, Italy \\ Mario Manassero * and Mirella Sansoni \\ Centro del C.N.R. per lo studio della sintesi e della struttura dei composti dei metalli di transizione, \\ via G. Venezian 21, 20133 Milano, Italy
}

Reaction of $\left[\mathrm{CO}_{3}(\mathrm{CO})_{9} \mathrm{CCl}\right]$ with $\left[\mathrm{Ni}_{6}(\mathrm{CO})_{12}\right]^{2-}$ results in a complicated mixture of mixed $\mathrm{Co}-\mathrm{Ni}$ carbide carbonyl clusters, among which the $\left[\mathrm{Co}_{3} \mathrm{Ni}_{9} \mathrm{C}(\mathrm{CO})_{20}\right]^{3-}$ trianion has been isolated in a pure crystalline state and fully characterized by $X$-ray crystallography. The metal framework of this compound is unprecedented in cluster geometries and may be described as a square antiprism of metal atoms tetra-capped on two alternate pairs of adjacent triangular faces. Despite the presence of a caged carbon atom in the square-antiprismatic cavity, the compound is readily degraded by carbon monoxide $\left(25^{\circ} \mathrm{C}\right.$, $1 \mathrm{~atm})$ mainly to a mixture of $\left[\mathrm{Co}(\mathrm{CO})_{4}\right]^{-}$and $\left[\mathrm{Ni}(\mathrm{CO})_{4}\right]$. Corresponding degradation of the cluster under a mixture of carbon monoxide and hydrogen yields, in addition, trace amounts of organics, mainly $C_{1}$ and $C_{2}$ hydrocarbons, probably derived from the carbide atom.

Homometallic cobalt carbide clusters, e.g. $\left[\mathrm{Co}_{6} \mathrm{C}(\mathrm{CO})_{15}\right]^{2-}$ and $\left[\mathrm{Co}_{6} \mathrm{C}(\mathrm{CO})_{14}\right]^{-1},{ }^{1,2}$ have been synthesized by condensation of $\left[\mathrm{Co}(\mathrm{CO})_{4}\right]^{-}$onto $\left[\mathrm{Co}_{3}(\mathrm{CO})_{9} \mathrm{CCl}\right]$. We have investigated a related synthesis of mixed $\mathrm{Co}^{-} \mathrm{Ni}$ carbide clusters. Owing to the lack of a readily available nickel carbonyl anionic species of low nuclearity, we have successfully employed the $\left[\mathrm{Ni}_{6}\right.$ $\left.(\mathrm{CO})_{12}\right]^{2-}$ dianion: elimination of $\mathrm{CO}$, as required by the condensation of the two reagents into higher nuclearity clusters, has been obtained through partial loss of nickel as $\left[\mathrm{Ni}(\mathrm{CO})_{4}\right]$.

The condensation between $\left[\mathrm{CO}_{3}(\mathrm{CO})_{9} \mathrm{CCl}\right]$ and $\left[\mathrm{Ni}_{6}\right.$ $\left.(\mathrm{CO})_{12}\right]^{2-}$ afforded several mixed $\mathrm{Co}-\mathrm{Ni}$ carbide clusters, whose structural and chemical characterization is currently underway. Here, we report the synthesis, structural characterization, and chemical behaviour of the first of such species which has been fully characterized by $X$-ray crystallography, namely $\left[\mathrm{Co}_{3} \mathrm{Ni}_{9} \mathrm{C}(\mathrm{CO})_{20}\right]^{3-}$ in its tetraethylammonium salt.

Prior to this work, mixed $\mathrm{Fe}^{-} \mathrm{Ni}$ carbide clusters, e.g. $\left[\mathrm{Fe}_{4} \mathrm{Ni}_{2} \mathrm{C}(\mathrm{CO})_{14}\right]^{2-}, \quad\left[\mathrm{Fe}_{5} \mathrm{NiC}(\mathrm{CO})_{16}\right]$, and $\left[\mathrm{Fe}_{5} \mathrm{NiC}-\right.$ $\left.(\mathrm{CO})_{15}\right]^{2-}, 3.4$ in which nickel plays only a secondary role in stabilizing the metal cage of the carbon atom, have been synthesized by polyhedral expansion of either $\left[\mathrm{Fe}_{4} \mathrm{C}(\mathrm{CO})_{12}\right]^{2-}$ or $\left[\mathrm{Fe}_{5} \mathrm{C}(\mathrm{CO})_{14}\right]^{2-}$ using $\left[\mathrm{Ni}(\operatorname{cod})_{2}\right](\mathrm{cod}=$ cyclo-octa-1,5diene).

\section{Results and Discussion}

Synthesis and Chemical Behaviour of $\left[\mathrm{Co}_{3} \mathrm{Ni}_{9} \mathrm{C}(\mathrm{CO})_{20}\right]^{3-}$ The $\left[\mathrm{Co}_{3} \mathrm{Ni}_{9} \mathrm{C}(\mathrm{CO})_{20}\right]^{3-}$ trianion is obtained by the slow addition (under a nitrogen atmosphere) of a tetrahydrofuran (thf) solution of $\left[\mathrm{CO}_{3}(\mathrm{CO})_{9} \mathrm{CCl}\right]$ to a stirred thf solution of $\left[\mathrm{NEt}_{4}\right]_{2}\left[\mathrm{Ni}_{6}(\mathrm{CO})_{12}\right]$, up to a 1:2 molar ratio. The formal stoicheiometry of the reaction is given by equation (1). However, under these experimental conditions, the condensation of

$$
\begin{aligned}
& 2\left[\mathrm{Ni}_{6}(\mathrm{CO})_{12}\right]^{2-}-\left[\mathrm{Co}_{3}(\mathrm{CO})_{9} \mathrm{CCl}\right] \rightarrow \\
& \quad\left[\mathrm{CO}_{3} \mathrm{Ni}_{6} \mathrm{Cr}(\mathrm{CO})_{20}\right]^{3-}+\mathrm{Cl}^{-}+3\left[\mathrm{Ni}(\mathrm{CO})_{4}\right]+\mathrm{CO}
\end{aligned}
$$

+ Carbidohexadeca- $\mu$-carbonyl-tetracarbonyl-polyhedro-nonanickeltricobaltate( $3-$ ).

Supplementary data arailable (No. SUP 23881, 21 pp.): structure factors, $\mathrm{H}$-atom co-ordinates, isotropic and anisotropic thermal parameters. See Instructions for Authors, J. Chem. Soc., Dalton Trans., 1984. Issue 1, pp. xvii-xix.

Non-S.I. unit employed: $\mathrm{atm}=101325 \mathrm{~N} \mathrm{~m}^{-2}$.
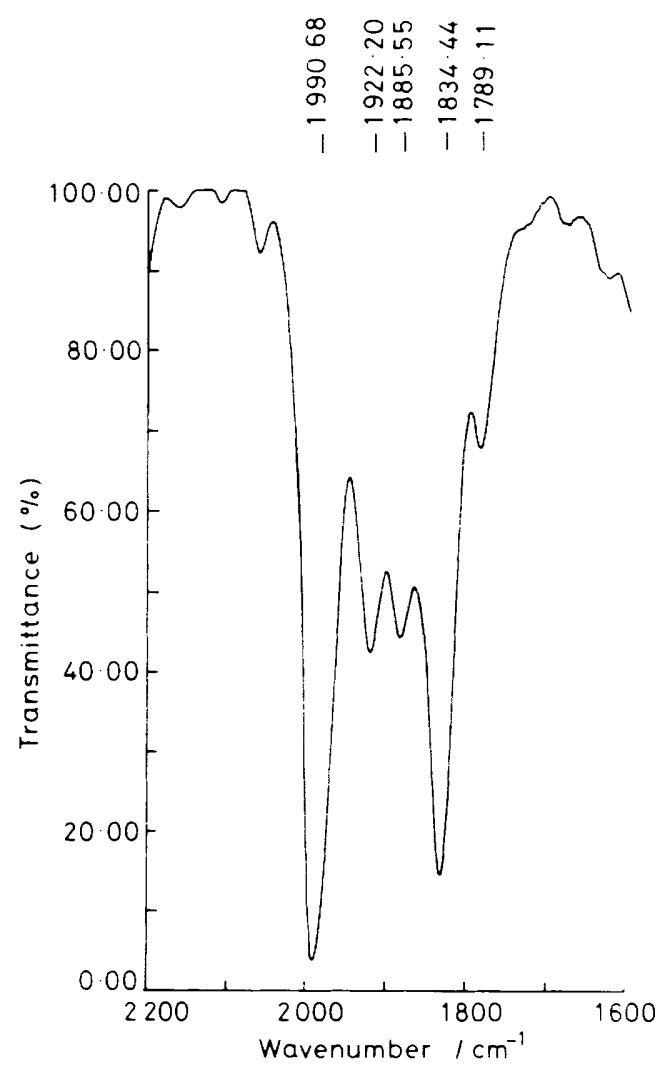

Figure 1. Intrared spectrum of $\left[\mathrm{NEt}_{4}\right]_{3}\left[\mathrm{CO}_{3} \mathrm{Ni}_{9} \mathrm{C}(\mathrm{CO})_{2} ..\right]$ in acetonitrile solution

$\left[\mathrm{Ni}_{6}(\mathrm{CO})_{12}\right]^{2-}$ onto $\left[\mathrm{CO}_{3}(\mathrm{CO})_{9} \mathrm{CCl}\right]$ may be more complicated than represented by equation (1). Thus, i.r. monitoring of the solution during reaction also shows the formation of minor amounts of $\left[\mathrm{Co}(\mathrm{CO})_{4}\right]^{-}$and a yet uncharacterized green anionic species. This last compound presents i.r. absorptions at $1995 \mathrm{~s}, 1985 \mathrm{~s}(\mathrm{sh}), 1950(\mathrm{sh})$, and $1840 \mathrm{~m}$, br $\mathrm{cm}^{-1}$, and appears to be a mixed $\mathrm{Co}^{-} \mathrm{Ni}$ carbide cluster on the basis of elemental analyses $(\mathrm{Co}: \mathrm{Ni}=1: 1.48)$.

The $\left[\mathrm{CO}_{3} \mathrm{Ni}_{9} \mathrm{C}(\mathrm{CO})_{20}\right]^{3-}$ trianion has been isolated as its 


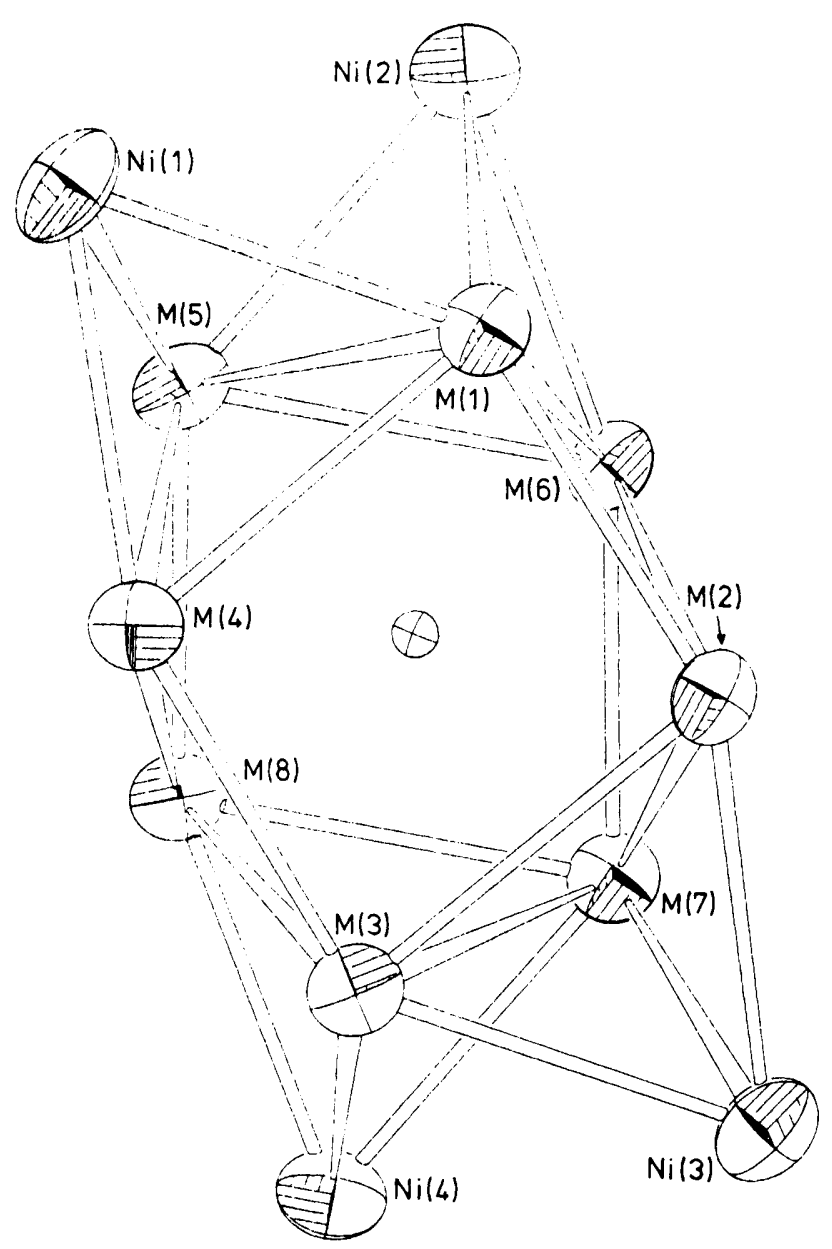

Figure 2. The metal skeleton with numbering of the $\left[\mathrm{Co}_{3} \mathrm{Ni}_{9} \mathrm{C}\right.$ $\left.(\mathrm{CO})_{20}\right]^{3-}$ trianion

tetraethylammonium salt by evaporation in vacuum of the reaction solution, followed by extraction with acetone of the residue, and precipitation by slow diffusion of isopropyl alcohol. Well shaped dark red crystals of $\left[\mathrm{NEt}_{4}\right]_{3}\left[\mathrm{Co}_{3} \mathrm{Ni}_{9} \mathrm{C}\right.$ (CO) ${ }_{20}$ ] were obtained in $\mathrm{ca} .50-60 \%$ yield. In its $\left[\mathrm{NEt}_{4}\right]^{+}$ salt the $\left[\mathrm{Co}_{3} \mathrm{Ni}_{9} \mathrm{C}(\mathrm{CO})_{20}\right]^{3-}$ trianion is sparingly soluble in the and $\operatorname{Pr}^{\prime} \mathrm{OH}$, and insoluble in non-polar solvents; on the other hand, it is fairly soluble in acetone and acetonitrile giving redbrown solutions which show i.r. absorptions in the carbonyl stretching region at $1990 \mathrm{~s}, 1922 \mathrm{~ms}, 1885 \mathrm{~ms}, 1834 \mathrm{~s}$, and $1789 \mathrm{w} \mathrm{cm}^{-1}$ (Figure 1). Elemental analysis of the crystals shows a $\left[\mathrm{NEt}_{4}\right]^{+}: \mathrm{Co}: \mathrm{Ni}$ ratio very close to $1: 1: 3$. The correct stoicheiometry and structure of the compound has been ascertained through a combination of elemental analysis and an $X$-ray structural determination (see Experimental section).

The salts of the $\left[\mathrm{CO}_{3} \mathrm{Ni}_{9} \mathrm{C}(\mathrm{CO})_{20}\right]^{3-}$ trianion are air sensitive both in the solid state and in solution. In solution, and under a carbon monoxide atmosphere $\left(25^{\circ} \mathrm{C}, 1 \mathrm{~atm}\right)$ the cluster is completely degraded by $\mathrm{CO}$ to a mixture of $\left[\mathrm{Co}(\mathrm{CO})_{4}\right]^{-}$and $\left[\mathrm{Ni}(\mathrm{CO})_{4}\right]$, according to reaction (2). This result is partially

$$
\begin{aligned}
& {\left[\mathrm{Co}_{3} \mathrm{Ni}_{9} \mathrm{C}(\mathrm{CO})_{20}\right]^{3-}+} 28 \mathrm{CO} \longrightarrow \\
& 9\left[\mathrm{Ni}(\mathrm{CO})_{4}\right]+3\left[\mathrm{Co}(\mathrm{CO})_{4}\right]^{-}+\mathrm{C}
\end{aligned}
$$

surprising because the presence of a caged carbon atom has been suggested to confer extra stability, with respect to degradation reactions by carbon monoxide, to cluster com-

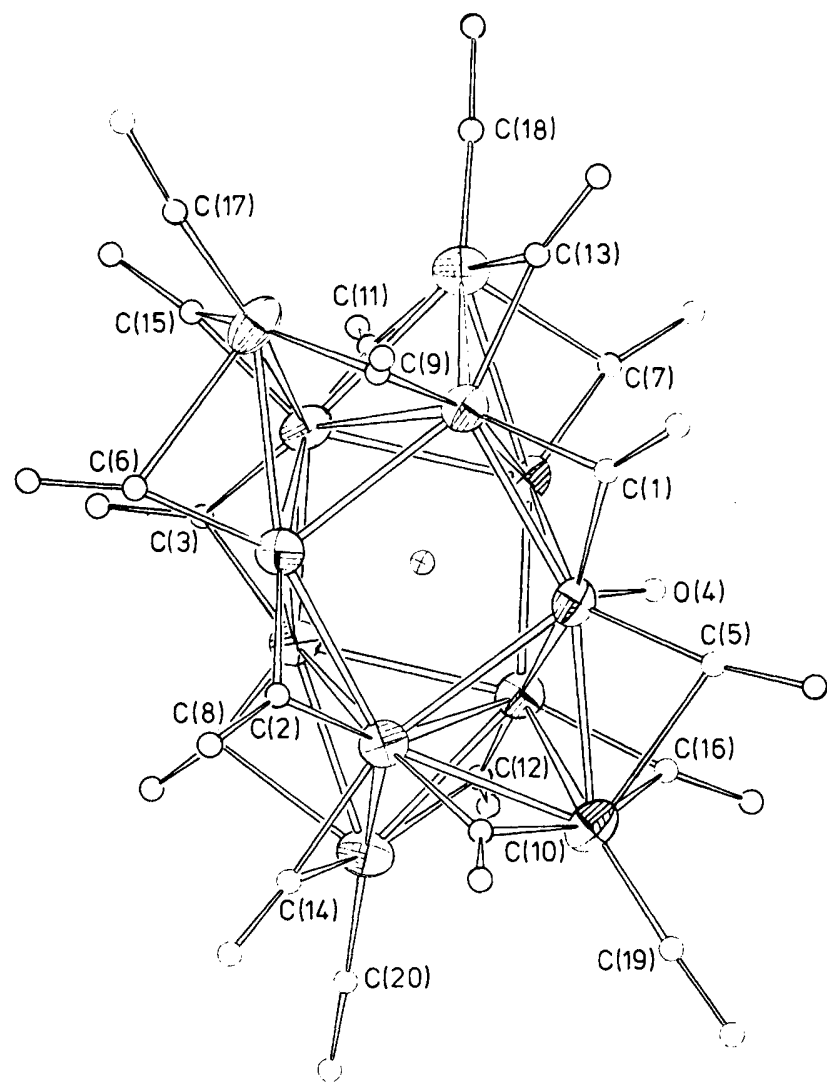

Figure 3. Whole structure of the $\left[\mathrm{Co}_{3} \mathrm{Ni}_{9} \mathrm{C}(\mathrm{CO})_{20}\right]^{3-}$ trianion; carbon and oxygen atoms are shown as small circles for the sake of clarity

pounds of the first transition metal series. ${ }^{5}$ Reaction (2) is probably a consequence of the fortuitous presence in this cluster of the same number of free negative charges and cobalt atoms, and is favoured by the well known stability of both $\left[\mathrm{Co}(\mathrm{CO})_{4}\right]^{-}$and $\left[\mathrm{Ni}(\mathrm{CO})_{4}\right]$. In spite of many efforts, we have so far been unable to establish the fate of the carbide carbon atom in reaction (2).

When the $\left[\mathrm{CO}_{3} \mathrm{Ni}_{9} \mathrm{C}(\mathrm{CO})_{20}\right]^{3-}$ trianion reacts in acetonitrile with a 1:1 mixture of hydrogen and carbon monoxide $\left(25{ }^{\circ} \mathrm{C}, 1 \mathrm{~atm}\right)$ complete degradation to $\left[\mathrm{Ni}(\mathrm{CO})_{4}\right]$ and $\left[\mathrm{Co}(\mathrm{CO})_{4}\right]^{-}$still occurs, and gas-chromatographic analysis of the gas phase showed the presence of traces of methane, ethylene, and ethane. The $C_{2}$ fraction accounts for over $80 \%$ of the organic mixture. The most probable origin for the $\mathrm{C}_{1}$ and $\mathrm{C}_{2}$ hydrocarbons is the interstitial carbide atom; however, we have not yet been able to prove unequivocally their origin through isotopic tracer studies.

The possible relationship between the observation of a relatively high fraction of $\mathrm{C}_{2}$ hydrocarbons and the recent isolation and characterization of bimetallic dicarbide clusters, such as $\left[\mathrm{Co}_{3} \mathrm{Ni}_{7} \mathrm{C}_{2}(\mathrm{CO})_{15}\right]^{3-}$ and $\left[\mathrm{Co}_{6} \mathrm{Ni}_{2} \mathrm{C}_{2}(\mathrm{CO})_{16}\right]^{2-}$, is at present under investigation. These two last compounds derive chemically from $\left[\mathrm{Co}_{3} \mathrm{Ni}_{9} \mathrm{C}(\mathrm{CO})_{20}\right]^{3-}$, and both contain an interstitial $\mathrm{C}_{2}$ fragment showing an interatomic $\mathrm{C}-\mathrm{C}$ separation of 1.41 and $1.49 \AA$, respectively. ${ }^{6,7}$

X-Ray Crystal Structure of $\left[\mathrm{Co}_{3} \mathrm{Ni}_{9} \mathrm{C}(\mathrm{CO})_{20}\right]^{3-}$. - The crystal structure of $\left[\mathrm{NEt}_{4}\right]_{3}\left[\mathrm{Co}_{3} \mathrm{Ni}_{9} \mathrm{C}(\mathrm{CO})_{20}\right]$ consists of the packing of ciscrete $\left[\mathrm{NEt}_{4}\right]^{+}$and $\left[\mathrm{Co}_{3} \mathrm{Ni}_{9} \mathrm{C}(\mathrm{CO})_{20}\right]^{3-}$ ions. Positional parameters are reported in Table 1 and selected bond distances and angles in Table 2. 
Table 1. Final positional parameters $\left(\times 10^{4}\right)$ with estimated standard deviations in parentheses

\begin{tabular}{|c|c|c|c|c|c|c|c|}
\hline Atom & $X / a$ & $Y / b$ & $Z / c$ & Atom & $X / a$ & $Y / b$ & $Z / c$ \\
\hline$M(1)$ & $2230(1)$ & $1695(1)$ & $4114(1)$ & $O(15)$ & $-1028(9)$ & $3735(8)$ & $3723(2)$ \\
\hline$M(2)$ & $3902(1)$ & $1747(1)$ & $3869(1)$ & $C(16)$ & $5623(12)$ & $3517(12)$ & $3692(4)$ \\
\hline$M(3)$ & $3199(1)$ & $2313(1)$ & $3229(1)$ & $O(16)$ & $6466(10)$ & $3921(9)$ & $3788(3)$ \\
\hline$M(4)$ & $1526(1)$ & $1943(1)$ & $3447(1)$ & $C(17)$ & $-886(14)$ & $1263(14)$ & $3969(4)$ \\
\hline$M(5)$ & $1290(1)$ & $3541(1)$ & $3874(1)$ & $O(17)$ & $-1650(12)$ & $800(11)$ & $4020(3)$ \\
\hline$M(6)$ & $3254(1)$ & $3515(1)$ & $4202(1)$ & $C(18)$ & $1190(15)$ & $3363(14)$ & $4879(4)$ \\
\hline$M(7)$ & $4160(1)$ & $3720(1)$ & $3692(1)$ & $O(18)$ & $857(11)$ & $3537(11)$ & $5142(3)$ \\
\hline$M(8)$ & $2211(1)$ & $3993(1)$ & $3384(1)$ & $C(19)$ & $6273(14)$ & $1879(13)$ & $3285(4)$ \\
\hline $\mathrm{Ni}(1)$ & $289(1)$ & $1836(1)$ & $3897(1)$ & $O(19)$ & $7034(11)$ & $1622(10)$ & $3169(3)$ \\
\hline $\mathrm{Ni}(2)$ & $1689(1)$ & $3100(1)$ & $4506(1)$ & $C(20)$ & $4273(15)$ & $4863(14)$ & $2760(4)$ \\
\hline $\mathrm{Ni}(3)$ & $5138(1)$ & $2242(1)$ & $3451(1)$ & $O(20)$ & $4652(11)$ & $5365(11)$ & $2543(3)$ \\
\hline $\mathrm{Ni}(4)$ & $3770(1)$ & $4107(1)$ & $3055(1)$ & $\mathrm{N}(1)$ & $-1674(9)$ & $3114(9)$ & $2211(3)$ \\
\hline C & $2748(9)$ & $2807(9)$ & $3725(3)$ & C(111) & $-774(13)$ & $2288(12)$ & $2163(4)$ \\
\hline$C(1)$ & $3338(12)$ & $695(12)$ & $4131(4)$ & $\mathrm{C}(112)$ & $270(16)$ & $2879(15)$ & $2068(5)$ \\
\hline$O(1)$ & $3624(9)$ & $-142(8)$ & $4270(2)$ & $\mathrm{C}(121)$ & $-1337(13)$ & $4003(13)$ & $2490(4)$ \\
\hline$C(2)$ & $1940(11)$ & $1705(11)$ & $3003(3)$ & $C(122)$ & $-966(16)$ & $3558(15)$ & $285 I(5)$ \\
\hline$O(2)$ & $1543(8)$ & $1388(8)$ & $2717(2)$ & $C(131)$ & $-2000(14)$ & $3752(13)$ & $1866(4)$ \\
\hline$C(3)$ & $1137(11)$ & $4739(11)$ & $3566(3)$ & $C(132)$ & $-2364(17)$ & $3027(16)$ & $1536(5)$ \\
\hline$O(3)$ & $588(8)$ & $5492(8)$ & $3503(2)$ & $C(141)$ & $-2598(13)$ & $2410(13)$ & $2308(4)$ \\
\hline C(4) & $4456(13)$ & $4317(12)$ & $4149(4)$ & $C(142)$ & $-3591(16)$ & $3066(15)$ & $2378(5)$ \\
\hline$O(4)$ & $5066(9)$ & $4928(8)$ & $4305(3)$ & $N(2)$ & $7285(9)$ & $2472(9)$ & $5023(3)$ \\
\hline$C(5)$ & $5193(12)$ & $1230(12)$ & $3956(4)$ & $C(211)$ & $6564(18)$ & $1417(17)$ & $4993(5)$ \\
\hline$O(5)$ & $5968(9)$ & $769(8)$ & $4087(3)$ & $C(212)$ & $5337(16)$ & $1616(15)$ & $4917(5)$ \\
\hline$C(6)$ & $326(12)$ & $1252(12)$ & $3334(4)$ & $C(221)$ & $7245(18)$ & $2960(17)$ & $4645(6)$ \\
\hline$O(6)$ & $-378(10)$ & $737(9)$ & $3180(3)$ & $C(222)$ & $7984(18)$ & $3879(17)$ & $4619(5)$ \\
\hline$C(7)$ & $3417(13)$ & $3743(12)$ & $4650(4)$ & $C(231)$ & $6881(18)$ & $3301(17)$ & $5270(5)$ \\
\hline$O(7)$ & $3764(9)$ & $4017(9)$ & $4940(3)$ & $C(232)$ & $6835(17)$ & $2964(16)$ & $5639(5)$ \\
\hline C(8) & $1976(11)$ & $4516(11)$ & $2952(3)$ & $C(241)$ & $8429(20)$ & $2151(20)$ & $5193(6)$ \\
\hline$O(8)$ & $1550(9)$ & $4822(8)$ & $2685(2)$ & $C(242)$ & $8969(16)$ & $1327(16)$ & $4967(5)$ \\
\hline$C(9)$ & $1262(13)$ & $615(12)$ & $4058(4)$ & $C(312)$ & $3552(32)$ & $2564(29)$ & $746(10)$ \\
\hline$O(9)$ & $1078(10)$ & $-324(9)$ & $4079(3)$ & $C(322)$ & $2856(27)$ & $921(26)$ & $1750(8)$ \\
\hline$C(10)$ & $4026(12)$ & $1150(12)$ & $3233(4)$ & $C(332)$ & $3456(26)$ & $3924(25)$ & $1726(8)$ \\
\hline$O(10)$ & $4172(10)$ & $240(9)$ & $3151(3)$ & $C(342)$ & $664(27)$ & $2532(25)$ & $938(9)$ \\
\hline C(11) & $1187(12)$ & $4404(11)$ & $4257(4)$ & $N(3)$ & $2665(11)$ & $2429(11)$ & $1271(4)$ \\
\hline$O(11)$ & $903(9)$ & $5267(9)$ & $4343(3)$ & $C(311)$ & $3002(32)$ & $2952(31)$ & $975(8)$ \\
\hline$C(12)$ & $4083(11)$ & $5044(10)$ & $3482(3)$ & $C(321)$ & $3283(30)$ & $1430(24)$ & $1400(9)$ \\
\hline$O(12)$ & $4185(8)$ & $5971(8)$ & $3520(2)$ & $C(331)$ & $2565(32)$ & $3172(26)$ & $1583(7)$ \\
\hline$C(13)$ & $2249(13)$ & $1640(13)$ & $4600(4)$ & $C(341)$ & $1427(22)$ & $2000(30)$ & $1153(10)$ \\
\hline$O(13)$ & $2477(10)$ & $1138(9)$ & $4852(3)$ & C(351) & $3008(51)$ & ( 773(49) & $951(12)$ \\
\hline$C(14)$ & $3533(12)$ & $2780(11)$ & $2798(4)$ & $C(361)$ & $2439(45)$ & $1447(43)$ & $1536(13)$ \\
\hline$O(14)$ & $3556(10)$ & $2510(9)$ & $2508(3)$ & $C(371)$ & $3634(42)$ & $2992(43)$ & $1454(14)$ \\
\hline$C(15)$ & $-197(12)$ & $3282(11)$ & $3801(4)$ & $C(381)$ & $1717(39)$ & $3019(45)$ & $1175(16)$ \\
\hline
\end{tabular}

The metal framework of $\left.\left[\mathrm{Co}_{3} \mathrm{Ni} \mathrm{C}_{9} \mathrm{CO}\right)_{20}\right]^{3-}$ is shown in Figure 2, and may be described as a distorted square antiprism of metal atoms which is tetra-capped on two alternate pairs of adjacent triangular faces. Major deviations from this description arise from the two square faces being more accurately described as two parallelograms having alternate angles of $c a .100$ and $79^{\circ}$, and two opposite edges elongated by ca. $0.17 \AA$ with respect to the other two; the four elongated edges (average $2.583 \AA$ ) correspond to the four capped interlayer triangular faces, while the four shorter contacts (mean $2.415 \AA$ ) are spanned by edge-bridging carbonyl groups. The eight interlayer $\mathrm{M}-\mathrm{M}$ bonds of the square-antiprismatic moiety have a mean value of $2.633 \AA$, derived from averaging alternately shorter and longer contacts of 2.580 and $2.687 \AA$ respectively, whereas the bonds connecting the four caps to the central $\mathrm{M}_{8}$ fragment show an average value of $2.465 \AA$. Such a metal polyhedron of statistical (see later) $222\left(D_{2}\right)$ idealized symmetry has no precedent in molecular cluster geometries, since all reported examples of clusters possessing a square-antiprismatic moiety are $\mu_{4}$ rather than $\mu_{3}$ capped, e.g. $\left[\mathrm{Ni}_{9} \mathrm{C}(\mathrm{CO})_{1-}\right]^{2-},{ }^{7} \quad\left[\mathrm{Rh}_{9} \mathrm{P}(\mathrm{CO})_{21}\right]^{2-},{ }^{8} \quad\left[\mathrm{Rh}_{10} \mathrm{E}(\mathrm{CO})_{22}\right]^{3-}$ $(E=P$ or As $),{ }^{9.10}$ and $\left[\mathrm{Rh}_{10} \mathrm{~S}(\mathrm{CO})_{22}\right]^{2-} .{ }^{11}$

An ORTEP drawing of the whole structure of the $\left[\mathrm{Co}_{3} \mathrm{Ni}_{9}-\right.$ $\left.\mathrm{C}(\mathrm{CO})_{20}\right]^{3-}$ trianion is reported in Figure 3.
The 12 metal atoms of $\left[\mathrm{Co}_{3} \mathrm{Ni}_{9} \mathrm{C}(\mathrm{CO})_{20}\right]^{3-}$ may be divided into three sets, each of them comprising four stereochemically equivalent metal atoms, with respect to metal and ligand coordination [viz. $\mathrm{M}(1)-\mathrm{M}(3)-\mathrm{M}(5)-\mathrm{M}(7), \mathrm{M}(2)-\mathrm{M}(4)-\mathrm{M}(6)^{-}$ $\mathrm{M}(8)$, and $\mathrm{Ni}(1)-\mathrm{Ni}(2)-\mathrm{Ni}(3)-\mathrm{Ni}(4)]$. Since elemental analysis of this diamagnetic compound shows a $\mathrm{Co}: \mathrm{Ni}$ ratio of $1: 3$, this implies a random distribution of cobalt and nickel atoms in the cluster framework. The four capping atoms, however, show a stereochemistry very close to that found around the unique nickel atom of the recently characterized $\left[\mathrm{Fe}_{3} \mathrm{Ni}\right.$ (CO) $\left.{ }_{12} \mathrm{H}\right]^{-.12}$ In the lack of direct crystallographic evidence, it seems conceivable that those atoms could be labelled as nickel atoms. Such a labelling would limit the random distribution of cobalt and nickel atoms to the square-antiprismatic moiety, and would allow the three cobalt atoms of the parent $\left[\mathrm{Co}_{3}\right.$ $\left.(\mathrm{CO})_{9} \mathrm{CCl}\right]$ to remain co-ordinated to the interstitial carbide atom in $\left[\mathrm{Co}_{3} \mathrm{Ni}_{9} \mathrm{C}(\mathrm{CO})_{20}\right]^{3-}$. A random distribution of cobalt and nickel atoms has been previously found in the octahedral $\left[\mathrm{Co}_{4} \mathrm{Ni}_{2}(\mathrm{CO})_{14}\right]^{2-}$ dianion. ${ }^{13}$

The distorted square-antiprismatic moiety cages the carbide atom. The $\mathrm{M}-\mathrm{C}$ (carbide) bonding interactions (Table 2) may be divided into two sets of four, which show average $\mathrm{M}-\mathrm{C}$ bond distances of 2.016 and $2.165 \AA$, respectively. Therefore, in common with the previously reported $\left[\mathrm{Co}_{8} \mathrm{C}(\mathrm{CO})_{18}\right]^{2-, 14}$ 
Table 2. Selected bond distances $(\AA)$ and angles $(")$ in the anion

\begin{tabular}{|c|c|c|c|c|c|c|c|}
\hline$M(1)-M(2)$ & $2.421(3)$ & $M(2)-M(6)$ & $2.722(3)$ & $\mathrm{M}(3)-\mathrm{Ni}(4)$ & $2.459(3)$ & $\mathrm{M}(5)-\mathrm{Ni}(2)$ & $2.455(3)$ \\
\hline$M(1)-M(4)$ & $2.590(3)$ & $M(2)-M(7)$ & $2.572(2)$ & $\mathrm{M}(4)-\mathrm{M}(5)$ & $2.612(3)$ & $\mathrm{M}(6)-\mathrm{M}(7)$ & $2.414(3)$ \\
\hline $\mathrm{M}(1)-\mathrm{M}(5)$ & \multirow{2}{*}{$\begin{array}{l}2.674(2) \\
2.594(2)\end{array}$} & $\mathrm{M}(2)-\mathrm{Ni}(3)$ & $2.464(3)$ & $M(4)-M(8)$ & $2.707(2)$ & $M(6)-N i(2)$ & $2.478(3)$ \\
\hline$M(1)-M(6)$ & & \multirow{2}{*}{$\begin{array}{l}\mathrm{M}(3)-\mathrm{M}(4) \\
\mathrm{M}(3)-\mathrm{M}(7)\end{array}$} & $2.415(3)$ & $\mathrm{M}(4)-\mathrm{Ni}(1)$ & $2.484(3)$ & $\mathrm{M}(7)-\mathrm{M}(8)$ & $2.573(2)$ \\
\hline $\mathrm{M}(1)-\mathrm{Ni}(1)$ & $2.459(3)$ & & $2.646(2)$ & $\mathrm{M}(5)-\mathrm{M}(6)$ & $2.594(2)$ & $\mathrm{M}(7)-\mathrm{Ni}(3)$ & $2.459(3)$ \\
\hline$M(1)-N i(2)$ & $2.459(3)$ & $M(3)-M(8)$ & $2.540(3)$ & \multirow{2}{*}{$\begin{array}{l}M(5)-M(8) \\
M(5)-N i(1)\end{array}$} & $2.408(3)$ & $\mathrm{M}(7)-\mathrm{Ni}(4)$ & $2.461(3)$ \\
\hline$M(2)-M(3)$ & $2.573(3)$ & $\mathrm{M}(3)-\mathrm{Ni}(3)$ & $2.456(3)$ & & $2.468(3)$ & $\mathrm{M}(8)-\mathrm{Ni}(4)$ & $2.482(3)$ \\
\hline$M(1)-C$ & \multirow{2}{*}{$\begin{array}{l}2.196(13) \\
1.973(12)\end{array}$} & $M(3)-C$ & $2.149(13)$ & 1 & $2.192(13)$ & $\mathrm{M}(7)-\mathrm{C}$ & $2.121(13)$ \\
\hline$M(2)-C$ & & $M(4)-C$ & $2.036(12)$ & $\mathrm{C}$ & $2.040(12)$ & $M(8)-C$ & $2.014(12)$ \\
\hline$M(1)-C(1)$ & $1.857(16)$ & $M(4)-C(6)$ & $1.731(16)$ & $-C(3)$ & $1.852(15)$ & $\mathrm{Ni}(2)-\mathrm{C}(18)$ & $1.673(20)$ \\
\hline$M(1)-C(9)$ & $1.800(16)$ & $M(5)-C(3)$ & $1.890(14)$ & $C(8)$ & $1.758(14)$ & $\mathrm{Ni}(3)^{-}$ & $2.296(16)$ \\
\hline$M(1)-C(13)$ & $1.856(17)$ & $M(5)-C(1))$ & $1.834(15)$ & $\cdots C(12)$ & $2.663(14)$ & $\mathrm{Ni}(3)-$ & $2.032(15)$ \\
\hline$M(2)-C(1)$ & $1.850(16)$ & $M(5)-C(15)$ & $1.873(13)$ & $-C(6)$ & $2.278(16)$ & $\mathrm{Ni}(3)$ & $1.886(15)$ \\
\hline$M(2)-C(5)$ & $1.728(16)$ & $M(6)-C(4)$ & $1.843(17)$ & ) $-\mathrm{C}(9)$ & $1.985(15)$ & $\mathrm{Ni}(3)$ & $1.704(18)$ \\
\hline$M(2) \cdots C(10)$ & $2.570(16)$ & $M(6)-C(7)$ & $1.716(16)$ & $-C(15)$ & $1.913(15)$ & $\mathrm{Ni}(4$ & $2.282(15)$ \\
\hline $\mathrm{M}(3)-\mathrm{C}(2)$ & $1.845(14)$ & $M(7)-C(4)$ & $1.887(16)$ & $C(17)$ & 19) & $\mathrm{Nic}$ & $1.998(14)$ \\
\hline$M(3)-C(10)$ & $1.776(16)$ & $\mathrm{M}(7)-\mathrm{C}(12)$ & $1.825(14)$ & 2) $-C(7)$ & $2.299(16)$ & $\mathrm{Nic}$ & $1.916(15)$ \\
\hline$M(3)-C(14)$ & $1.852(17)$ & $M(7)-C(16)$ & $1.852(16)$ & $C(11)$ & $1.935(14)$ & $\mathrm{Ni}(4)-\mathrm{C}(20)$ & $1.662(19)$ \\
\hline$M(4)-C(2)$ & $1.872(15)$ & & $\mathrm{Ni}($ & 2) $-C(13)$ & $1.956(16)$ & & \\
\hline (1) & & $D(6)$ & 1.1 & & & & 20) \\
\hline$C(2)$ & .2011 & $C(7)-O(7)$ & (19) & $(12)$ & & $\mathrm{Cl}$ & $1.159(24)$ \\
\hline $\mathrm{C}(3)-\mathrm{O}(3)$ & $.163(17)$ & $\mathrm{C}(8)-\mathrm{O}(8)$ & $1.148(17)$ & $O(13) 1$. & & $(18)$ & $3(25)$ \\
\hline (4) & .174 & $\mathrm{C}(9)-\mathrm{O}(9)$ & $1.194(19)$ & $D(14) 1$. & & (19) & $1.155(24)$ \\
\hline$C(5$ & .175 & $C(10)-O(10)$ & $1.193(20)$ & $-O(15) 1$. & 9) & $\mathrm{C}(20$ & $1.192(24)$ \\
\hline$M(2)$ & & & $1)-M(4)-M$ & ( (1) & Mi 6 & $4(8)$ & $81.6(1)$ \\
\hline$-M(3$ & & & b) $-M(5)-1$ & $31.3(1)$ & $\mathrm{M}($ & 7) & $98.4(1)$ \\
\hline $\mathbf{M}(2)-\mathbf{M}(3)-\mathbf{M}(4$ & & & C) 1 (1) & $077(1)$ & & & \\
\hline$M(1)-C(1)-M(2$ & & 等 & $\mathrm{M}(4)-\mathrm{C}(6)-\mathrm{O}(6)$ & $163.1(1.5)$ & $\mathrm{Ni}(2$ & 111 & $134.7(1.2)$ \\
\hline$M(1$ & & 1.4) & $\mathrm{Ni}(1)-\mathrm{C}(6$ & $121.7(1.3)$ & Mo & & $80.0(5)$ \\
\hline 1) $-\mathrm{O}(1)$ & & $7(1.4)$ & $(7)-\mathrm{Ni}(2)$ & $74.7(5)$ & $\mathrm{Mi}$ & 2) & $146.9(1.2)$ \\
\hline$(2)-M(4$ & & $0(6)$ & $\mathrm{M}(6)-\mathrm{C}(7)-\mathrm{O}(7)$ & $163.3(1.5)$ & $\mathrm{Ni}($ & 12) & $132.9(1.1)$ \\
\hline $\mathrm{M}(3)-\mathrm{C}(2)-\mathrm{O}(2)$ & 140 & $5(1.3)$ & $\mathrm{Ni}(2)-\mathrm{C}(7)-\mathrm{O}(7)$ & $121.9(1.3)$ & $M(1)$ & ii $(2)$ & $80.3(6)$ \\
\hline $\mathrm{M}(4)-\mathrm{C}(2)-\mathrm{O}(2)$ & 138 & $3(1.2)$ & $M(8)-C(8)-N i(4)$ & $74.5(5)$ & $\mathbf{M}(1$ & & $145.6(1.5)$ \\
\hline$-\mathrm{M}(8$ & & $1(5)$ & $M(8)-$ & $162.1(1.4)$ & $\mathrm{Ni}(2$ & 3) & $(1.4)$ \\
\hline$(3)-O(3)$ & & $6(1.3)$ & $(8)-O(8)$ & $122.9(1.2)$ & $M(3$ & & $81.5(6)$ \\
\hline (3) $\mathrm{O}(3)$ & 139 & $9(1.2)$ & $M(1)-C(9)-N i(1)$ & $80.9(6)$ & $M(3)$ & 14) & $143.1(1.3)$ \\
\hline$(4)^{-}-M(7$ & & $7(6)$ & $\mathrm{M}(1)-\mathrm{C}(9)-\mathrm{O}(9)$ & $148.5(1.3)$ & $\mathrm{Ni}(4)$ & $9(14)$ & $135.3(1.3)$ \\
\hline$(4)-O(4)$ & & $9(1.4$ & $\mathrm{Ni}(1)-\mathrm{C}(9)-\mathrm{O}(9)$ & $130.6(1.3)$ & $M(3$ & & $81.3(6)$ \\
\hline$-\mathrm{O}(4)$ & & .4( & (10)-Ni(3) & $80.0(6)$ & $M(5)^{-}$ & & $141.0(1.2)$ \\
\hline 5) $-\mathrm{Ni}(3$ & & $.0(5)$ & $\mathrm{M}(3)-\mathrm{C}(10)-\mathrm{O}(10)$ & $151.5(1.3)$ & $\mathrm{Ni}(1)$ & $\partial(15)$ & $137.5(1.2)$ \\
\hline (5) $-O(5)$ & 162 & $9(1.5)$ & $\mathrm{Ni}(3)-\mathrm{C}(10)-\mathrm{O}(10)$ & $128.0(1.2)$ & $M(7)-$ & $\mathrm{Ni}(3)$ & $82.3(6)$ \\
\hline$(5)-O(5)$ & 123 & $1(1.3)$ & $\mathrm{M}(5)-\mathrm{C}(11)-\mathrm{Ni}(2)$ & $81.3(6)$ & $\mathrm{M}(7)-$ & & $141.8(1.3)$ \\
\hline $\mathrm{M}(4)-\mathrm{C}(6)-\mathrm{Ni}(1$ & & & $\mathrm{M}(5)-\mathrm{C}(11)-\mathrm{O}(11)$ & $143.8(1.2)$ & $\mathrm{Ni}(3)-$ & 6) & $135.9(1.4)$ \\
\hline & $\mathrm{Ni}(1)-\mathrm{Cl}$ & $O(17)$ & $174.9(1.6)$ & 7 & $O(19)$ & 179. & \\
\hline & $\mathrm{Ni}(2)-\mathrm{C}($ & 3) & $178.8(1.6)$ & He & $O(20)$ & $177.1(1.7)$ & \\
\hline & $M(2)-C^{-}$ & & $105.0(5)$ & $\mathrm{M}(6)-\mathrm{C}-\mathrm{M}$ & $4(8)$ & $107.1(5)$ & \\
\hline
\end{tabular}

there is present a distinct preference of the carbide atom for tetrahedral co-ordination with four out of the eight metal atoms. It seems likely that attainment of such a tetrahedral stereochemistry by the carbide atom could be at the origin of some of the observed distortions of the $\mathrm{M}_{8}$ antiprismatic moiety of $\left[\mathrm{CO}_{3} \mathrm{Ni}_{4} \mathrm{C}(\mathrm{CO})_{20}\right]^{3-}$ such as, for instance, the rhombohedral deformation of the two square faces.

Skeletal electron-pair theories suggest that capping of a triangular face of an $N$-vertex metal polyhedron should not alter the number of filled molecular orbitals of the polyhedral moiety. ${ }^{15}$ The distorted square-antiprismatic anion $\left[\mathrm{Co}_{3} \mathrm{Ni}_{9} \mathrm{C}\right.$ $\left.(\mathrm{CO})_{20}\right]^{3-}$ has $6 \mathrm{~N}-10$ filled cluster valence molecular orbitals (c.v.m.o.s); i.e. one more than the tetragonally distorted square-antiprismatic $\left[\mathrm{Co}_{8} \mathrm{C}(\mathrm{CO})_{18}\right]^{2-}(6 \mathrm{~N}-9),{ }^{14}$ and one less than the $D_{4 i}$ square-antiprismatic $\left[\mathrm{Ni}_{3} \mathrm{C}(\mathrm{CO})_{16}\right]^{2-}(6 \mathrm{~N}-$
11). ${ }^{2}$ The latter is isoelectronic with the $D_{d d}$ square-antiprismatic $\mathrm{Bi}_{8}{ }^{2+}$ naked cluster. ${ }^{16}$ It appears, therefore, conceivable that distortions of a square-antiprismatic framework may be associated with a variation in the number of required filled c.v.m.o.s. A similar behaviour has been previously noticed in the $\mathrm{Ge}_{9}{ }^{2-}$ and $\mathrm{Ge}_{9}{ }^{4-}$ naked clusters. ${ }^{17}$

As shown in Figure 3, the 20 carbonyl ligands may be roughly classified into two sets: the first set comprises 16 edge-bridging carbonyl groups (labelled $1-16$ in Figure 3), while the second set comprises four purely terminal $(17-20)$ carbonyl groups bonded to the four capping atoms [Ni-C(av) $\because=1.684 ; \mathrm{C}-\mathrm{O}(\mathrm{av})=1.169 \AA]$. The 16 edge-bridging ligands may be further divided into four sets of four stereochemically equivalent carbonyl groups: thus, ligands labelled $1-4$ span the four shortest edges of the two rhombohedral faces of the $\mathrm{M}_{\mathbf{8}}$ central moiety, and are essentially symmetrical [M-C(av) 
$1.862 ; \mathrm{C}-\mathrm{O}(\mathrm{av})=1.184 \AA]$. On the contrary, ligands $5-16$ span all the edges connecting the four capping nickel atoms to the $\mathbf{M}_{\mathbf{8}}$ unit and show different degrees of asymmetry. Those labelled 5-8 are better described as semi-bridging, ${ }^{18}$ showing $\mathrm{M}-\mathrm{C}$ contacts much shorter than the corresponding $\mathrm{Ni}-\mathrm{C}$ contacts $[\mathrm{M}-\mathrm{C}(\mathrm{av})=1.733 ; \mathrm{Ni}-\mathrm{C}(\mathrm{av})=2.289 ; \mathrm{C}-\mathrm{O}(\mathrm{av})=$ $1.170 \AA]$. Those labelled $9-12$ may be defined as unsymmetrically bridging ligands $[\mathrm{M}-\mathrm{C}(\mathrm{av})=1.809 ; \mathrm{Ni}-\mathrm{C}(\mathrm{av})=1.988$; $\mathrm{C}-\mathrm{O}(\mathrm{av})=1.185 \AA]$, while the remaining four $(13-16)$ are only slightly unsymmetrical $[\mathrm{M}-\mathrm{C}(\mathrm{av})=1.858 ; \mathrm{Ni}-\mathrm{C}(\mathrm{av})=$ 1.918; $\mathrm{C}-\mathrm{O}(\mathrm{av})=1.168 \AA]$.

The complicated stereochemistry of the carbonyl groups in the solid-state structure of $\left[\mathrm{Co}_{3} \mathrm{Ni}{ }_{9} \mathrm{C}(\mathrm{CO})_{20}\right]^{3-}$ is probably the consequence of both the necessity to equalize the charge onto different metal atoms and of steric crowding among the $\mathrm{CO}$ ligands. However, in spite of such complicated stereochemistry, the i.r. spectrum of $\left[\mathrm{Co}_{3} \mathrm{Ni}_{9} \mathrm{C}(\mathrm{CO})_{20}\right]^{3-}$ in solution (Figure 1) is relatively simple, and shows equally intense absorption bands in the terminal- and bridging-carbonyl stretching region.

\section{Experimental}

Synthesis and Chemical Characterization of $\left[\mathrm{Co}_{3} \mathrm{Ni}_{9}-\right.$ $\left.\mathrm{C}(\mathrm{CO})_{20}\right]^{3-}$.- All operations were carried out under a nitrogen atmosphere and in anhydrous solvents. Infrared spectra were recorded on a Perkin-Elmer 457 grating spectrophotometer using calcium fluoride cells. Gas-chromatographic analyses were performed on a Perkin-Elmer SIGMA 115 gas chromatograph equipped with hot wire and flame ionisation detectors on both Porapak QS and Chromosorb 101 columns and making use of a metal-uncontaminated silica injector. Elemental analyses were obtained as described elsewhere, ${ }^{19}$ and $\left[\mathrm{Ni}_{6}(\mathrm{CO})_{12}\right]^{2-}$ and $\left[\mathrm{Co}_{3}(\mathrm{CO})_{9} \mathrm{CCl}\right]$ were synthesized by literature methods. ${ }^{9.20}$

Synthesis of $\left[\mathrm{NEt}_{4}\right]_{3}\left[\mathrm{Co}_{3} \mathrm{Ni}_{9} \mathrm{C}(\mathrm{CO})_{20}\right]$. $\left[\mathrm{NEt}_{4}\right]_{2}\left[\mathrm{Ni}_{6}(\mathrm{CO})_{12}\right]$ $(1.09 \mathrm{~g})$ was suspended under a nitrogen atmosphere in anhydrous th $f\left(30 \mathrm{~cm}^{3}\right)$ in a round-bottomed flask equipped with a dropping funnel. A solution of $\left[\mathrm{Co}_{3}(\mathrm{CO})_{9} \mathrm{CCl}\right](0.365 \mathrm{~g})$ in thf $\left(20 \mathrm{~cm}^{3}\right)$ was added dropwise through the dropping funnel and with stirring over a $2 \mathrm{~h}$ period. The resulting red-brown suspension was evaporated to dryness in a vacuum. The residue was washed twice with isopropyl alcohol $\left(10 \mathrm{~cm}^{3}\right)$ and extracted in acetone $\left(25 \mathrm{~cm}^{3}\right)$. By slow diffusion of this solution in isopropyl alcohol $\left(40 \mathrm{~cm}^{3}\right)$, well shaped crystals of $\left[\mathrm{NEt}_{4}\right]_{3}\left[\mathrm{Co}_{3} \mathrm{Ni}_{4} \mathrm{C}(\mathrm{CO})_{20}\right]$ were obtained. Yield $0.47 \mathrm{~g}$. The compound is soluble in acetone and acetonitrile, sparingly soluble in thf and alcohols, and insoluble in non-polar solvents such as toluene or $n$-heptane. \{Found: $\left[\mathrm{NEt}_{4}\right]^{+}, 22.95 ; \mathrm{Co}$, 10.70: $\mathrm{Ni} .30 .90$. $\left[\mathrm{NEt}_{4}\right]_{3}\left[\mathrm{Co}_{3} \mathrm{Ni}_{9} \mathrm{C}(\mathrm{CO})_{20}\right]$ requires $\left[\mathrm{NEt}_{4}\right]^{+}$ 23.40: $\mathrm{Co}, 10.60 . \mathrm{N}, 31.70^{\circ}$

Reaction of $\left[\mathrm{CO}_{3} \mathrm{Ni}_{4} \mathrm{C}(\mathrm{CO})_{20}\right]^{3-}$ with $\mathrm{CO}$ and $\mathrm{H}_{2}(1: 1)$. $\left[\mathrm{NEt}_{4}\right]_{3}\left[\mathrm{Co}_{3} \mathrm{Ni}_{4} \mathrm{C}(\mathrm{CO})_{20}\right](0.263 \mathrm{~g})$ was dissolved under nitrogen in acetonitrile $\left(5 \mathrm{~cm}^{3}\right.$ ) in a $50-\mathrm{cm}^{3}$ flask or glass pressure vessel. The resulting red-brown solution, showing i.r. absorptions at $1990 \mathrm{~s}, 1915 \mathrm{~m}, 1830 \mathrm{~ms}$, and $1770 \mathrm{w} \mathrm{cm} \mathrm{cm}^{-1}$, upon stirring under a carbon monoxide and hydrogen $(1: 1)$ atmosphere at room temperature. turned initially brown and then bzcame lighter. I.r. monitoring of the solution showed i.r. absorptions at 2040 and $1890 \mathrm{~cm}$, due to $\left[\mathrm{Ni}(\mathrm{CO})_{4}\right]$ and [Co$\left.(\mathrm{CO})_{4}\right]^{-}$respectively. The gas phase of the reaction, as well as the original $\mathrm{CO}-\mathrm{H}_{2}$ mixture, were analysed by gas chromatography under strictly identical conditions. On comparison of these. the spectrum after the gas phase reaction shows the additional presence of $C_{1}$ and $C_{2}$ hydrocarbons with the $C_{2}$ fration accounting for over $80^{\circ}$ of the organic products.
$\mathrm{X}-$ Ray Intensity Measurements.-Crystal data. $\mathrm{C}_{45} \mathrm{H}_{60} \mathrm{Co}_{3}-$ $\mathrm{N}_{3} \mathrm{Ni}_{9} \mathrm{O}_{20}, M=1668.1$, deep red monoclinic prisms, space group $P 2_{1} / c$ (no. 14) from systematic absences, $a=12.543(3)$, $b=12.404(5), c=38.249(9) \AA, \beta=98.53(3)^{\circ}, U=5885.6$ $\AA^{3}, D_{\mathrm{m}}=1.91(3), D_{\mathrm{c}}=1.882 \mathrm{~g} \mathrm{~cm}^{-3}$ for $Z=4, \mu\left(\mathrm{Mo}-K_{\alpha}\right)=$ $37.2 \mathrm{~cm}^{-1}, \lambda\left(\mathrm{Mo}-K_{x}\right)=0.71069 \AA$. The unit-cell parameters were obtained from a least-squares refinement of $\theta$ values, measured on the diffractometer, for 32 reflections in the range $13<2 \theta<33^{\circ}$.

A specimen of maximum dimensions $0.50 \times 0.30 \times 0.35$ $\mathrm{mm}$, bound by faces $001,00 \overline{1}, 10 \overline{2}, \overline{1} 02,012,015,0 \overline{1} 2,0 \overline{1} 2$, was mounted on the BASIC diffractometer ${ }^{21}$ with the $c$ axis approximately parallel to $z$. Intensities were measured for 10500 reflections (corresponding to the $=h-k-l$ quadrant in the $2 \theta$ range $6.5-50^{\circ}$ ) using the $\omega$-scan technique, with a scan range of $1^{\circ}$ and a scan speed of $2.5^{\circ} \mathrm{min}^{-1}$. Backgrounds were counted for $12 \mathrm{~s}$ each, at the extreme points of the scan range. Periodical monitoring of these reflections revealed an average linear decay of the diffracted intensity which reached a maximum of $c a .4 \%$ at the end of data collection. Structure factors were computed by application of Lorentz, polarization, decay, and absorption corrections. The latter was evaluated by the numerical integration method, using a $8 \times 8 \times 8$ Gaussian grid, ${ }^{22}$ only for those reflections having $I \geqslant \sigma(I)$ to save computing time. Transmission factors were found in the range $0.74-0.26$. Averaging of equivalent reflections resulted in a total of 5411 independent structure amplitudes, of which 4040 having $I \geqslant 3 \sigma(l)$ were used for structure solution and refinement.

Structure solution and refinement. The structure was solved by direct methods, using MULTAN. The $E$ map from the best set showed 12 peaks of similar height, which were all refined, at this stage, as $\mathrm{Ni}$ atoms. The resulting $R$ was 0.25 and the subsequent iterative application of difference-Fourier syntheses and blocked-matrix least-squares refinement allowed the whole structure to be revealed. One of the tetraethylammonium cations [labelled $\mathrm{N}(3), C(3 j k)$ in Table 1] was found to be partially disordered: the four methylenic carbon atoms appeared in the Fourier map to be split into eight halfatoms, approximately defining two tetrahedra around the unique $\mathrm{N}(3)$ atom. These eight peaks [labelled $\mathrm{C}(311)$ $\mathrm{C}(381)]$ were forced to occupy the vertices of two regular tetrahedra (of edge $2.48 \AA$ ), which were refined as rigid bodies with an overall thermal factor each. The corresponding methylic carbon atoms $[\mathrm{C}(3 j 2)]$ did not appear as distinctly split into pairs, despite being affected by high thermal parameters (see Supplementary publication). In the final stage of refinement, the eight metal atoms defining the irregular squareantiprismatic moiety were given an atomic scattering factor equal to $\frac{5}{8} f_{\mathrm{Ni}}-\frac{3}{8} f_{\mathrm{Co}}$ (see Results and Discussion section). The anomalous dispersion of $\mathrm{Ni}$ and $\mathrm{Co}$ was taken into account in both their real and imaginary components. Final weights were given according to the formula $w=\left[\sigma_{F_{0}}^{2}+\right.$ $\left.\left(A \cdot F_{\mathrm{o}}\right)^{2}\right]^{-1}$, with $A=0.035$, thus obtaining a fairly constant distribution of $u\left(F_{0}-F_{\mathrm{c}}\right)^{2}$ as a function of $F_{\mathrm{o}}$. The final agreement indices were $R=0.055$ and $R^{\prime}=0.074$, where $R^{\prime}=\left[\sum w\left(F_{\mathrm{o}}-k F_{\mathrm{c}} \mid\right)^{2 / \sum w} F_{\mathrm{o}}{ }^{2}\right]^{\frac{1}{2}}$. The hydrogen atoms of cations 1 and 2 were introduced in their postulated positions $\left(d_{C-H}=1.0 \AA\right.$, staggered conformation), and not refined. Each was assigned a thermal parameter $1.0 \AA^{2}$ higher than that of the carbon atom to which it is bonded.

Atomic scattering factors were taken from ref. 23 for the non-hydrogen atoms, and from ref. 24 for the hydrogen atoms; $\Delta f^{\prime}$ and $\Delta f^{\prime \prime}$ for $\mathrm{Ni}$ and $\mathrm{Co}$ were taken from ref. 25 . The final difference Fourier did not show peaks exceeding 0.5 e $\AA^{-3}$. All computations were carried out on a UNIVAC $1100 / 80$ computer. Local programs were used, with the exception of MULTAN for direct methods and ORTEP for drawings. 


\section{References}

1 V. G. Albano, D. Braga, P. Chini, G. Ciani, and S. Martinengo, J. Chem. Soc., Dalton Trans., 1982, 645.

2 V. G. Albano, P. Chini, G. Ciani, M. Sansoni, and S. Martinengo, J. Chem. Soc., Dalton Trans., 1980, 163.

3 M. Tachikawa, A. C. Sievert, E. L. Muetterties, R. R. Thompson, C. S. Day, and V. W. Day, J. Am. Chem. Soc., 1980, 102, 1725 .

4 M. Tachikawa, R. L. Geerts, and E. L. Muetterties, J. Organomet. Chem., 1981, 213, 11.

5 P. Chini, G. Longoni, and V. G. Albano, Adt. Organomet. Chem., 1976, 14, 285.

6 A. Arrigoni, A. Ceriotti, R. Della Pergola, G. Longoni, M. Manassero, N. Masciocchi, and M. Sansoni, Angew. Chem. in the press.

7 G. Longoni, A. Ceriotti, R. Della Pergola, M. Manassero, M. Perego, G. Piro, and M. Sansoni, Philos. Trans. R. Soc. London, Ser. $A, 1982,308,47$.

8 J. L. Vidal, W. E. Walker, R. L. Pruett, and R. C. Schoening, Inorg. Chem., 1979, 18, 129.

9 J. L. Vidal, W. E. Walker, and R. C. Schoening, Inorg. Chem., $1981,20,238$.

10 J. L. Vidal, Inorg. Chem., 1981, 20, 243.

11 G. Ciani, L. Garlaschelli, A. Sironi, and S. Martinengo, $J$. Chem. Soc., Chem. Commun., 1981, 563.

12 A. Ceriotti, P. Chini, A. Fumagalli, T. F. Koetzle, G. Longoni, and F. Takusagawa, Inorg. Chem., in the press.
13 V. G. Albano, G. Ciani, and P. Chini, J. Chem. Soc., Dalton Trans., 1974, 432.

14 V. G. Albano, P. Chini, G. Ciani, S. Martinengo, and M. Sansoni, J. Chem. Soc., Dalton Trans., 1978, 463.

15 D. G. Evans and D. P. M. Mingos, Organometallics, 1983, 2, 435 and refs. therein.

16 B. Krebs, M. Hucke, and C. J. Brendel, Angew. Chem., Int. Ed. Engl., 1982, 21, 445.

17 C. H. Belin, J. D. Corbett, and A. Cisar, J. Am. Chem. Soc., 1977, 99, 7163.

18 F. A. Cotton, Prog. Inorg. Chem., 1974, $21,1$.

19 G. Longoni, P. Chini, and A. Cavalieri, Inorg. Chem., 1976, 15, 3025.

20 R. Ercoli, E. Santambrogio, and G. Tettamanti Casagrande, Chim. Ind. (Milan), 1962, 44, 1344.

21 L. Casella, M. Gullotti, A. Pasini, G. Ciani, M. Manassero, and A. Sironi, Inorg. Chim. Acta, 1978, 26, L1.

22 W. R. Busing and H. A. Levy, Acta Crystallogr., 1957, 10, 180.

23 D. T. Cromer and J. B. Mann, Acta Crystallogr., Sect. A, 1968, 24, 321 .

24 J. B. Forsyth and M. Wells, Acta Crystallogr., 1959, 12, 412.

25 'International Tables for $X$-Ray Crystallography,' Kynoch Press, Birmingham, 1974, vol. 4.

Received 1st August 1983; Paper 3/1338 